\title{
On some properties of general aggregation operators based on a fuzzy equivalence relation
}

\author{
Pavels Orlovs $^{1}$ Svetlana Asmuss ${ }^{1}$ \\ ${ }^{1}$ Institute of Mathematics and Computer Science, University of Latvia
}

\begin{abstract}
Our paper deals with constructions of upper and lower general aggregation operators, which are based in their definitions on a fuzzy equivalence relation. We study some properties of these aggregation operators which are important in the context of aggregation of fuzzy sets. In particular we consider different types of monotonicity and show that obtained results allow us to describe approximate system based on the constructed operators.
\end{abstract}

Keywords: Aggregation operator, general aggregation operator, fuzzy equivalence relation, upper and lower operators

\section{Introduction}

In this paper we develop the concept of upper and lower general aggregation operators with respect to a fuzzy equivalence relation $E$. This concept was introduced by the authors in $[15,16]$. Now we are focusing on different properties of these aggregation operators, which allows us to enrich existing framework. In our previous works $[13,14]$ we studied general aggregation operators based on a crisp equivalence relation instead of $E$. The idea was to aggregate fuzzy sets in accordance with classes of equivalence generated by this crisp equivalence relation. Such construction appeared while we were approaching an analysis of optimal solution for bilevel linear programming problems. In that case a crisp equivalence relation was induced by an upper level objective function.

Taking into account that fuzzy equivalence relations represent the fuzzification of equivalence relations and extensional fuzzy subsets play the role of fuzzy equivalence classes, we consider upper and lower general aggregation operators in the context of extensional fuzzy sets. It is important that the output of upper and lower general aggregation operators corresponding to fuzzy equivalence $E$ are extensional with respect to $E$. In some cases while aggregating extensional fuzzy sets it could be necessary to obtain as a result an extensional fuzzy set as well, but an ordinary general aggregation does not ensure this property.

In this paper we consider different properties of upper and lower general aggregation operators. Some of them deals with preservation of properties of an ordinary aggregation operator. We also study the monotonicity of these operators with respect to an aggregation operator, a t-norm and a fuzzy equivalence relation. We show that satisfaction of these properties ensures the construction of an approximate system based on upper and lower general aggregation operators. The concept of an approximate system was introduced and studied in $[5,7,18]$. Among the most important examples of approximate systems are approximate systems induced by fuzzy equivalence relations. These approximate systems are related to fuzzy rough sets, see e.g. [4].

\section{Preliminaries}

In this section first we will remind the definitions of an ordinary aggregation operator as well as general aggregation operator, which acts on fuzzy structures. As the examples of widely used aggregation operators we can mention the arithmetic and geometric means, the minimum and maximum operators, t-norms, t-conorms and others. Let us start with the classical notion of an aggregation operator (see, e.g., $[1,3,6]$ ).

Definition 2.1 A mapping $A: \bigcup_{n}[0,1]^{n} \rightarrow[0,1]$ is called an aggregation operator if the following conditions hold:

(A1) $A(0, \ldots, 0)=0$;

(A2) $A(1, \ldots, 1)=1$;

(A3) for all $n \in \mathbb{N}$ and for all $t_{1}, \ldots, t_{n} \in[0,1]$,

$$
\tau_{1}, \ldots, \tau_{n} \in[0,1]:
$$

$$
\begin{gathered}
t_{1} \leq \tau_{1}, \ldots, t_{n} \leq \tau_{n} \Longrightarrow \\
\Longrightarrow A\left(t_{1}, \ldots, t_{n}\right) \leq A\left(\tau_{1}, \ldots, \tau_{n}\right) .
\end{gathered}
$$

Conditions (A1) and (A2) are called the boundary conditions of $A,(\mathrm{~A} 3)$ means the monotonicity of $A$.

The general aggregation operator $\tilde{A}$ acting on $[0,1]^{X}$, where $[0,1]^{X}$ is the set of all fuzzy subsets of a set $X$, was introduced in 2003 by A. Takaci [19]. Let us note that for simplicity we do not distinguish between fuzzy sets and their membership functions. We denote an order on $[0,1]^{X}$ by $\preceq$. The least and the greatest elements of this order are denoted by $\tilde{0}$ and $\tilde{1}$, which are indicators of $\varnothing$ and $X$ respectively, i.e.

$$
\tilde{0}(x)=0 \text { and } \tilde{1}(x)=1 \text { for all } x \in X \text {. }
$$


Definition 2.2 A mapping

$$
\tilde{A}: \bigcup_{n}\left([0,1]^{X}\right)^{n} \rightarrow[0,1]^{X}
$$

is called a general aggregation operator if the following conditions hold:

$(\tilde{A} 1) \tilde{A}(\tilde{0}, \ldots, \tilde{0})=\tilde{0}$;

$(\tilde{A} 2) \tilde{A}(\tilde{1}, \ldots, \tilde{1})=\tilde{1}$;

( $\tilde{A} 3)$ for all $n \in \mathbb{N}$ and for all $\mu_{1}, \ldots, \mu_{n} \in[0,1]^{X}$, $\eta_{1}, \ldots, \eta_{n} \in[0,1]^{X}$ :

$$
\begin{aligned}
& \mu_{1} \preceq \eta_{1}, \ldots, \mu_{n} \preceq \eta_{n} \Longrightarrow \\
\Longrightarrow & \tilde{A}\left(\mu_{1}, \ldots, \mu_{n}\right) \preceq \tilde{A}\left(\eta_{1}, \ldots, \eta_{n}\right) .
\end{aligned}
$$

We consider the case:

$\mu \preceq \eta$ if and only if $\mu(x) \leq \eta(x)$ for all $x \in X$, for $\mu, \eta \in[0,1]^{X}$.

Let us note that a specific case is the aggregation of a singleton. Scientists often propose the following conventions (see e.g. [6]): $A(x)=x$ for all $x \in X$ (in the case of aggregation) and $\tilde{A}(\mu)=\mu$ for all $\mu \in[0,1]^{X}$ (in the case of general aggregation). Throughout this paper we will not follow these conventions. Moreover, for the suggested constructions of upper and lower general aggregation operators (see Definition 3.1) equality $\widetilde{A}(\mu)=\mu$ does not fulfil in general case. As a result of the proposed aggregation (upper or lower) we always obtain an extensional fuzzy set (see Definition 3.2). Thereby, if argument $\mu$ is not an extensional fuzzy set, then this equality does not hold.

There exist several approaches to construct a general aggregation operator $\tilde{A}$ based on an ordinary aggregation operator $A$. The most simplest one is the pointwise extension of an aggregation operator A:

$$
\tilde{A}\left(\mu_{1}, \ldots, \mu_{n}\right)(x)=A\left(\mu_{1}(x), \ldots, \mu_{n}(x)\right),
$$

where $\mu_{1}, \ldots, \mu_{n} \in[0,1]^{X}$ are fuzzy sets and $x \in X$.

A widely used approach to constructing a general aggregation operator $\tilde{A}$ is the $T$ - extension [19], which idea comes from the classical extension principle and uses a $t$-norm $T$ (see, e.g., [10]):

$$
\begin{gathered}
\tilde{A}\left(\mu_{1}, \ldots, \mu_{n}\right)(x)= \\
=\sup _{x=A\left(x_{1}, \ldots, x_{n}\right)} T\left(\mu_{1}\left(x_{1}\right), \ldots, \mu_{n}\left(x_{n}\right)\right) .
\end{gathered}
$$

Here $\mu_{1}, \ldots, \mu_{n} \in[0,1]^{X}$ and $x, x_{1} \ldots, x_{n} \in X$, where $X=[0,1]$.

We introduce another construction of a general aggregation operator, based on a left-continuous $t$ norm $T$, the corresponding residuum $\vec{T}$ and a $T$ fuzzy equivalence relation $E$. Let us recall the basic definitions.

Fuzzy equivalence relations were introduced in 1971 by L.A. Zadeh [21] for the strongest $t$-norm $T_{M}$ (minimum t-norm) and later were developed and applied by several authors in more general cases (see e.g. [11]).
Definition 2.3 Let $T$ be a $t$-norm and $E$ be a fuzzy relation on a set $X$, i.e. $E$ is a fuzzy subset of $X \times X$. A fuzzy relation $E$ is a $T$-fuzzy equivalence relation if for all $x, y, z \in X$ it holds

(E1) $E(x, x)=1$ (reflexivity);

(E2) $E(x, y)=E(y, x)$ (symmetry);

(E3) $T(E(x, y), E(y, z)) \leq E(x, z)$ (T-transitivity).

Definition 2.4 Let $T$ be a left continuous t-norm. The residuum $\vec{T}$ of $T$ is defined for all $x, y \in[0,1]$ by

$$
\vec{T}(x \mid y)=\sup \{\alpha \in[0,1] \mid T(\alpha, x) \leq y\} .
$$

We recall the following basic properties of the residuum, which will be used later in the paper:

$(\vec{T} 1) \vec{T}(x \mid y)=1$ if and only if $x \leq y$;

$(\vec{T} 2) \vec{T}(1 \mid y)=y$

$(\vec{T} 3) \vec{T}(0 \mid y)=1$;

$(\vec{T} 4)$ if $y=0$ and $x \neq 0$, then $\vec{T}(x \mid y)=0$;

$(\vec{T} 5)$ the residuum is a non-increasing function with respect to the first argument and a nondecreasing function with respect to the second argument:

$$
\begin{gathered}
x_{1} \leq x_{2} \Longrightarrow \vec{T}\left(x_{1} \mid y\right) \geq \vec{T}\left(x_{2} \mid y\right) ; \\
y_{1} \leq y_{2} \Longrightarrow \vec{T}\left(x \mid y_{1}\right) \leq \vec{T}\left(x \mid y_{2}\right) ; \\
(\vec{T} 6) \quad T(z, x) \leq y \Longleftrightarrow z \leq \vec{T}(x \mid y) \\
\text { (here } \left.x, x_{1}, x_{2}, y, y_{1}, y_{2}, z \in[0,1]\right) .
\end{gathered}
$$

\section{Upper and lower general aggregation operators}

Now we give the definition of upper and lower general aggregation operators, based on a leftcontinuous $t$-norm $T$, the corresponding residuum $\vec{T}$ and a $T$-fuzzy equivalence relation $E$.

Definition 3.1 Let $A: \bigcup_{n}[0,1]^{n} \rightarrow[0,1]$ be an aggregation operator, $T$ be a left continuous t-norm, $\vec{T}$ be the residuum of $T$ and $E$ be a $T$-fuzzy equivalence relation defined on a set $X$. The upper and lower general aggregation operators $\tilde{A}_{E, T}$ and $\tilde{A}_{E, \vec{T}}$ are defined respectively by

$$
\begin{gathered}
\tilde{A}_{E, T}\left(\mu_{1}, \ldots, \mu_{n}\right)(x)= \\
=\sup _{x^{\prime} \in X} T\left(E\left(x, x^{\prime}\right), A\left(\mu_{1}\left(x^{\prime}\right), \ldots, \mu_{n}\left(x^{\prime}\right)\right)\right), \\
\tilde{A}_{E, \vec{T}}\left(\mu_{1}, \ldots, \mu_{n}\right)(x)= \\
=\inf _{x^{\prime} \in X} \vec{T}\left(E\left(x, x^{\prime}\right) \mid A\left(\mu_{1}\left(x^{\prime}\right), \ldots, \mu_{n}\left(x^{\prime}\right)\right)\right),
\end{gathered}
$$

where $x \in X$ and $\mu_{1}, \ldots, \mu_{n} \in[0,1]^{X}$.

It is easy to show that conditions $(\tilde{A} 1),(\tilde{A} 2)$ and ( $\tilde{A} 3)$ are satisfied for such constructions. Thereby we obtained two general aggregation operators $\tilde{A}_{E, T}$ and $\tilde{A}_{E, \vec{T}}$. We could consider them as upper and 
lower approximations of a general aggregation operator $\tilde{A}$, which is the pointwise extension of an ordinary aggregation operator $A$. It is clear, that for all $\mu_{1}, \ldots, \mu_{n} \in[0,1]^{X}$ it holds

$$
\begin{gathered}
\tilde{A}_{E, \vec{T}}\left(\mu_{1}, \ldots, \mu_{n}\right) \leq \tilde{A}\left(\mu_{1}, \ldots, \mu_{n}\right) \leq \\
\leq \tilde{A}_{E, T}\left(\mu_{1}, \ldots, \mu_{n}\right) .
\end{gathered}
$$

The notions of upper and lower general aggregation operators in our works [13], [14] were preceded by a general aggregation operator based on a crisp equivalence relation instead of a fuzzy one. Let $\rho \in X \times X$ be a crisp equivalence relation defined on a set $X$. We take $E=E_{\rho}$, where

$$
E_{\rho}(x, y)= \begin{cases}1, & (x, y) \in \rho, \\ 0, & (x, y) \notin \rho,\end{cases}
$$

and for an ordinary aggregation operator $A$ obtain $\tilde{A}_{E_{\rho}, T}$ and $\tilde{A}_{E_{\rho}, \vec{T}}$ for any t-norm $T$ :

$$
\begin{gathered}
\tilde{A}_{E_{\rho}, T}\left(\mu_{1}, \ldots, \mu_{n}\right)(x)= \\
=\sup _{x^{\prime} \in X:\left(x^{\prime}, x\right) \in \rho} A\left(\mu_{1}\left(x^{\prime}\right), \ldots, \mu_{n}\left(x^{\prime}\right)\right), \\
\tilde{A}_{E_{\rho}, \vec{T}}\left(\mu_{1}, \ldots, \mu_{n}\right)(x)= \\
=\inf _{x^{\prime} \in X:\left(x^{\prime}, x\right) \in \rho} A\left(\mu_{1}\left(x^{\prime}\right), \ldots, \mu_{n}\left(x^{\prime}\right)\right) .
\end{gathered}
$$

The idea of these general aggregation operators is based on the classes of equivalence generated by a crisp equivalence relation $\rho$. In fact, we aggregate fuzzy sets in accordance with classes of equivalence generated by equivalence relation $\rho$. As a result we have the following property:

$$
\begin{gathered}
(x, y) \in \rho \Longrightarrow \\
\Longrightarrow \tilde{A}_{E_{\rho}, T}\left(\mu_{1}, \ldots, \mu_{n}\right)(x)=\tilde{A}_{E_{\rho}, T}\left(\mu_{1}, \ldots, \mu_{n}\right)(y), \\
\tilde{A}_{E_{\rho}, \vec{T}}\left(\mu_{1}, \ldots, \mu_{n}\right)(x)=\tilde{A}_{E_{\rho}, \vec{T}}\left(\mu_{1}, \ldots, \mu_{n}\right)(y)
\end{gathered}
$$

for all $x, y \in X$ and for all $\mu_{1}, \ldots, \mu_{n} \in[0,1]^{X}$. An analogue for this property in the case of $T$-fuzzy equivalence relation $E$ is the fact that the results of aggregations $\tilde{A}_{E, T}\left(\mu_{1}, \ldots, \mu_{n}\right)$ and $\tilde{A}_{E, \vec{T}}\left(\mu_{1}, \ldots, \mu_{n}\right)$ are extensional fuzzy sets with respect to $E$ for all $\mu_{1}, \ldots, \mu_{n} \in[0,1]^{X}$.

Definition 3.2 Let $T$ be a t-norm and $E$ be a $T$ fuzzy equivalence relation on a set $X$. A fuzzy subset $\mu \in[0,1]^{X}$ is called extensional with respect to $E$ if:

$$
T(E(x, y), \mu(y)) \leq \mu(x) \text { for all } x, y \in X .
$$

Extensional fuzzy subsets have been widely studied in the literature [2], [8], [9], [11]. We recall two approximation operators $\phi_{E}$ and $\psi_{E}$, which appear in a natural way in the theory of fuzzy rough sets (see, e.g., [4], [12], [20]). Fuzzy sets $\phi_{E}(\mu)$ and $\psi_{E}(\mu)$ were introduced to provide upper and lower approximation of a fuzzy set $\mu$ with respect to fuzzy equivalence relation $E[11]$.
Definition 3.3 Let $T$ be a left-continuous t-norm, $\vec{T}$ be it's residuum and $E$ be a $T$-fuzzy equivalence relation on a set $X$. The maps $\phi_{E}:[0,1]^{X} \rightarrow[0,1]^{X}$ and $\psi_{E}:[0,1]^{X} \rightarrow[0,1]^{X}$ are defined by

$$
\begin{aligned}
& \phi_{E}(\mu)(x)=\sup _{y \in X} T(E(x, y), \mu(y)), \\
& \psi_{E}(\mu)(x)=\inf _{y \in X} \vec{T}(E(x, y) \mid \mu(y)),
\end{aligned}
$$

for all $x \in X$ and for all $\mu \in[0,1]^{X}$.

It is known that for all $\mu \in[0,1]^{X}$ fuzzy sets $\phi_{E}(\mu)$ and $\psi_{E}(\mu)$ are extensional with respect to $E$. Let us note that we always obtain extensional fuzzy sets as results of the upper and lower general aggregation operators.

Proposition 3.4 Let $T$ be a left-continuous $t$ norm, $E$ be a T-fuzzy equivalence relation on a set $X$. Let $\tilde{A}_{E, T}$ and $\tilde{A}_{E, T}$ be upper and lower general aggregation operators. Then fuzzy sets $\tilde{A}_{E, T}\left(\mu_{1}, \ldots, \mu_{n}\right)$ and $\tilde{A}_{E, \vec{T}}\left(\mu_{1}, \ldots, \mu_{n}\right)$ are extensional with respect to $E$ for each $n \in \mathbb{N}$ and for all fuzzy sets $\mu_{1}, \ldots, \mu_{n} \in[0,1]^{X}$.

The concept of upper and lower general aggregation operators was introduced and studied in the context of extensional fuzzy sets in our previous works $[15,16]$.

\section{Properties derived from an ordinary aggregation operator}

In this section we study some of the most important properties of upper and lower general aggregation operators $\tilde{A}_{E, T}$ and $\tilde{A}_{E, \vec{T}}$ derived from the properties of ordinary aggregation operator $A$ (see $[1,3,6])$.

Symmetry. For all $n \in \mathbb{N}$ it holds: if for every permutation $\pi: N \rightarrow N$, where $N=\{1, \ldots, n\}$, and for all $t_{1}, \ldots, t_{n} \in[0,1]$

$$
A\left(t_{1}, \ldots, t_{n}\right)=A\left(t_{\pi(1)}, \ldots, t_{\pi(n)}\right),
$$

then for all $\pi: N \rightarrow N$ the following equalities

$$
\begin{aligned}
& \tilde{A}_{E, T}\left(\mu_{1}, \ldots, \mu_{n}\right)=\tilde{A}_{E, T}\left(\mu_{\pi(1)}, \ldots, \mu_{\pi(n)}\right), \\
& \tilde{A}_{E, \vec{T}}\left(\mu_{1}, \ldots, \mu_{n}\right)=\tilde{A}_{E, \vec{T}}\left(\mu_{\pi(1)}, \ldots, \mu_{\pi(n)}\right)
\end{aligned}
$$

hold for all $\mu_{1}, \ldots, \mu_{n} \in[0,1]^{X}$.

Associativity. Associativity of $A$ in general is not preserved by upper and lower general aggregation operators $\tilde{A}_{E, T}$ and $\tilde{A}_{E, \vec{T}}$. Let us provide an illustrative example for this fact.

For simplicity we consider the discrete two-point universe $X$, and we use a vector form for fuzzy sets and a matrix form for $T$-fuzzy equivalence relation. Let us take $T_{L}$-fuzzy equivalence relation $\left(T_{L}\right.$ is Lukasiewicz t-norm)

$$
E=\left(\begin{array}{cc}
1 & 0.7 \\
0.7 & 1
\end{array}\right)
$$


and fuzzy sets $\mu_{1}, \mu_{2}$ and $\mu_{3}$ :

$$
\mu_{1}=\left(\begin{array}{l}
0.4 \\
0.2
\end{array}\right), \mu_{2}=\left(\begin{array}{l}
0.3 \\
0.7
\end{array}\right), \mu_{3}=\left(\begin{array}{c}
0.1 \\
0.9
\end{array}\right)
$$

We obtain the following results for the upper general aggregation operator:

$$
\begin{gathered}
A \widetilde{V} G_{E, T_{L}}\left(A \widetilde{V} G_{E, T_{L}}\left(\mu_{1}, \mu_{2}\right), \mu_{3}\right)=\left(\begin{array}{c}
0.375 \\
0.675
\end{array}\right), \\
A \widetilde{V} G_{E, T_{L}}\left(\mu_{1}, A \widetilde{V} G_{E, T_{L}}\left(\mu_{2}, \mu_{3}\right)\right)=\left(\begin{array}{c}
0.45 \\
0.50
\end{array}\right),
\end{gathered}
$$

and the following results for the lower general aggregation operator:

$$
\begin{aligned}
& A \widetilde{V} G_{E, \vec{T}_{L}}\left(A \widetilde{V} G_{E, \vec{T}_{L}}\left(\mu_{1}, \mu_{2}\right), \mu_{3}\right)=\left(\begin{array}{c}
0.225 \\
0.525
\end{array}\right), \\
& A \widetilde{V} G_{E, \vec{T}_{L}}\left(\mu_{1}, A \widetilde{V} G_{E, \vec{T}_{L}}\left(\mu_{2}, \mu_{3}\right)\right)=\left(\begin{array}{c}
0.30 \\
0.35
\end{array}\right),
\end{aligned}
$$

where $A=A V G$ is an ordinary arithmetic mean aggregation operator. As one can see, associativity does not hold in both cases.

Now we will look at existence of absorbent, neutral and idempotent elements.

Absorbent element. Let $M_{a b}$ be a set of all absorbent elements (or annihilators) of ordinary aggregation operator $A$, i.e. for all $d \in M_{a b} \subset[0,1]$, for all $n \in \mathbb{N}$ and for all $t_{1}, \ldots, t_{n} \in[0,1]$

$$
A\left(t_{1}, \ldots, d, \ldots, t_{n}\right)=d .
$$

Then any fuzzy set $\tilde{d} \in[0,1]^{X}$, such that $\tilde{d}$ is extensional with respect to $E$ and $\tilde{d}(x) \in M_{a b}$ for all $x \in X$, is an absorbent element of $\tilde{A}_{E, T}$ and $\tilde{A}_{E, \vec{T}}$. Indeed, for all $n \in \mathbb{N}$ and for all $\mu_{1}, \ldots, \mu_{n} \in[0,1]^{X}$ we have

$$
\begin{gathered}
\tilde{A}_{E, T}\left(\mu_{1}, \ldots, \tilde{d}, \ldots, \mu_{n}\right)(x)= \\
=\sup _{x^{\prime} \in X} T\left(E\left(x, x^{\prime}\right), A\left(\mu_{1}\left(x^{\prime}\right), \ldots, \tilde{d}\left(x^{\prime}\right), \ldots, \mu_{n}\left(x^{\prime}\right)\right)\right)= \\
=\sup _{x^{\prime} \in X} T\left(E\left(x, x^{\prime}\right), \tilde{d}\left(x^{\prime}\right)\right)=\tilde{d}(x), \\
\tilde{A}_{E, \vec{T}}\left(\mu_{1}, \ldots, \tilde{d}, \ldots, \mu_{n}\right)(x)= \\
=\inf _{x^{\prime} \in X} \vec{T}\left(E\left(x, x^{\prime}\right) \mid A\left(\mu_{1}\left(x^{\prime}\right), \ldots, \tilde{d}\left(x^{\prime}\right), \ldots, \mu_{n}\left(x^{\prime}\right)\right)\right)= \\
=\inf _{x^{\prime} \in X} \vec{T}\left(E\left(x, x^{\prime}\right) \mid \tilde{d}\left(x^{\prime}\right)\right)=\tilde{d}(x) .
\end{gathered}
$$

Neutral element. Let $M_{n e}$ be a set of all neutral elements of ordinary aggregation operator $A$, i.e. for all $e \in M_{n e} \subset[0,1]$, for all $n \in \mathbb{N}$ and for all $t_{1}, \ldots, t_{n} \in[0,1]$

$$
\begin{aligned}
& A\left(t_{1}, \ldots, t_{i-1}, e, t_{i+1}, \ldots, t_{n}\right)= \\
& =A\left(t_{1}, \ldots, t_{i-1}, t_{i+1}, \ldots, t_{n}\right) .
\end{aligned}
$$

Then any fuzzy set $\tilde{e} \in[0,1]^{X}$, such that $\tilde{e}$ is extensional with respect to $E$ and $\tilde{e}(x) \in M_{n e}$ for all $x \in X$, is a neutral element of $\tilde{A}_{E, T}$ and $\tilde{A}_{E, T}$. Indeed, for all $n \in \mathbb{N}$ and for all $\mu_{1}, \ldots, \mu_{n} \in[0,1]^{X}$ we have

$$
\begin{gathered}
\tilde{A}_{E, T}\left(\mu_{1}, \ldots, \tilde{e}, \ldots, \mu_{n}\right)= \\
=\sup _{x^{\prime} \in X} T\left(E\left(x, x^{\prime}\right), A\left(\mu_{1}\left(x^{\prime}\right), \ldots, \tilde{e}\left(x^{\prime}\right), \ldots, \mu_{n}\left(x^{\prime}\right)\right)\right)= \\
=\sup _{x^{\prime} \in X} T\left(E\left(x, x^{\prime}\right), A\left(\mu_{1}\left(x^{\prime}\right), \ldots, \mu_{n}\left(x^{\prime}\right)\right)\right)= \\
=\tilde{A}_{E, T}\left(\mu_{1}, \ldots, \mu_{n}\right), \\
\tilde{A}_{E, \vec{T}}\left(\mu_{1}, \ldots, \tilde{e}, \ldots, \mu_{n}\right)= \\
=\inf _{x^{\prime} \in X} \vec{T}\left(E\left(x, x^{\prime}\right) \mid A\left(\mu_{1}\left(x^{\prime}\right), \ldots, \tilde{e}\left(x^{\prime}\right), \ldots, \mu_{n}\left(x^{\prime}\right)\right)\right)= \\
=\inf _{x^{\prime} \in X} \vec{T}\left(E\left(x, x^{\prime}\right) \mid A\left(\mu_{1}\left(x^{\prime}\right), \ldots, \mu_{n}\left(x^{\prime}\right)\right)\right)= \\
=\tilde{A}_{E, \vec{T}^{\prime}}\left(\mu_{1}, \ldots, \mu_{n}\right) .
\end{gathered}
$$

Idempotent element. Let $M_{i d}$ be a set of all idempotent elements of ordinary aggregation operator $A$, i.e. for all $t \in M_{i d} \subset[0,1]$ and for all $n \in \mathbb{N}$

$$
A(\overbrace{t, \ldots, t}^{n})=t
$$

Then any fuzzy set $\mu \in[0,1]^{X}$, such that $\mu$ is extensional with respect to $E$ and $\mu(x) \in M_{i d}$ for all $x \in X$, is an idempotent element of $\tilde{A}_{E, T}$ and $\tilde{A}_{E, \vec{T}}$. Indeed, for all $\mu_{1}, \ldots, \mu_{n} \in[0,1]^{X}$ we have

$$
\begin{gathered}
\tilde{A}_{E, T}(\overbrace{\mu, \ldots, \mu}^{n})(x)= \\
=\sup _{x^{\prime} \in X} T\left(E\left(x, x^{\prime}\right), A(\overbrace{\mu\left(x^{\prime}\right), \ldots, \mu\left(x^{\prime}\right)}^{n})\right)= \\
=\sup _{x^{\prime} \in X} T\left(E\left(x, x^{\prime}\right), \mu\left(x^{\prime}\right)\right)=\mu(x),
\end{gathered}
$$

and analogously

$$
\begin{gathered}
\tilde{A}_{E, \vec{T}}(\mu, \ldots, \mu)(x)= \\
=\inf _{x^{\prime} \in X} \vec{T}\left(E\left(x, x^{\prime}\right) \mid A\left(\mu\left(x^{\prime}\right), \ldots, \mu\left(x^{\prime}\right)\right)\right)= \\
=\inf _{x^{\prime} \in X} \vec{T}\left(E\left(x, x^{\prime}\right) \mid \mu\left(x^{\prime}\right)\right)=\mu(x) .
\end{gathered}
$$

Now we will illustrate the properties mentioned above with some particular ordinary aggregation operators $A$.

$\mathbf{A}=\min$. Taking $A=$ min we will obtain symmetric aggregation operators $\tilde{A}_{E, T}$ and $\tilde{A}_{E, T}$ with neutral element $\tilde{e}=\tilde{1}$ and absorbent element $\tilde{d}=\tilde{0}$. In this case all extensional fuzzy sets with respect to $E$ are idempotent elements for these operators.

$\mathbf{A}=\max$. Taking $A=\max$ we obtain operators with similar properties to the case, when $A=\min$ 
with a remark, that neutral and absorbent elements are $\tilde{e}=\tilde{0}$ and $\tilde{d}=\tilde{1}$ respectively.

$\mathbf{E}=\mathbf{E}_{\rho}$ In the case of crisp equivalence relation $\rho$ general aggregation operators $\tilde{A}_{E_{\rho}, T}$ and $\tilde{A}_{E_{\rho}, \vec{T}}$ inherit such properties of ordinary aggregation operator $A$ as symmetry, associativity, existence of absorbent, neutral and idempotent elements. Let us note that in this case fuzzy set $\mu$ is extensional with respect to $E_{\rho}$ if it holds

$$
\mu(x)=\mu(y) \Longleftrightarrow(x, y) \in \rho .
$$

\section{Different types of monotonicity for upper and lower general aggregation operators}

In this section we will look at monotonicity of upper and lower general aggregation operators $\tilde{A}_{E, T}$ and $\tilde{A}_{E, \vec{T}}$ with respect to ordinary aggregation operator $A$, t-norm $T$ and $T$-fuzzy equivalence relation $E$. Monotonicity with respect to inputs is ensured by the property $(\tilde{A} 3)$.

In order to consider monotonicity with respect to ordinary aggregation operator $A$, we assume that

$$
A^{1} \leq A^{2} \Longleftrightarrow A^{1}\left(t_{1}, \ldots, t_{n}\right) \leq A^{2}\left(t_{1}, \ldots, t_{n}\right)
$$

for all $t_{1}, \ldots, t_{n} \in[0,1]$. We also suppose that for all $\mu_{1}, \ldots, \mu_{n} \in[0,1]^{X}$

$$
\begin{gathered}
\tilde{A}_{E, T}^{1} \leq \tilde{A}_{E, T}^{2} \Longleftrightarrow \\
\Longleftrightarrow \tilde{A}_{E, T}^{1}\left(\mu_{1}, \ldots, \mu_{n}\right)(x) \leq \tilde{A}_{E, T}^{2}\left(\mu_{1}, \ldots, \mu_{n}\right)(x), \\
\tilde{A}_{E, T}^{1} \leq \tilde{A}_{E, \vec{T}}^{2} \Longleftrightarrow \\
\Longleftrightarrow \tilde{A}_{E, T}^{1}\left(\mu_{1}, \ldots, \mu_{n}\right)(x) \leq \tilde{A}_{E, \vec{T}}^{2}\left(\mu_{1}, \ldots, \mu_{n}\right)(x),
\end{gathered}
$$

$x \in X$.

As a result if it holds $A^{1} \leq A^{2}$, then $\tilde{A}_{E, T}^{1} \leq \tilde{A}_{E, T}^{2}$ and $\tilde{A}_{E, \vec{T}}^{1} \leq \tilde{A}_{E, \vec{T}}^{2}$.

Now we will consider monotonicity of upper and lower general aggregation operators $\tilde{A}_{E, T}$ and $\tilde{A}_{E, \vec{T}}$ with respect to a left-continuous t-norm $T$. It is easy to show, that if $T_{1} \leq T_{2}$, then $\tilde{A}_{E, T_{1}} \leq \tilde{A}_{E, T_{2}}$ and $A_{E, \vec{T}_{1}} \geq \tilde{A}_{E, \vec{T}_{2}}$.

To study monotonicity of $\tilde{A}_{E, T}$ and $\tilde{A}_{E, \vec{T}}$ with respect to a $T$-fuzzy equivalence relation $E$ we define the order between fuzzy equivalencde relations:

$$
E_{1} \leq E_{2} \Longleftrightarrow E_{1}(x, y) \leq E_{2}(x, y) \text { for all } x, y \in X
$$

Taking into account the monotonicity of $T$, we obtain that if $E_{1} \leq E_{2}$, then

$$
\begin{gathered}
\tilde{A}_{E_{1}, T}\left(\mu_{1}, \ldots, \mu_{n}\right)(x)= \\
=\sup _{x^{\prime} \in X} T\left(E_{1}\left(x, x^{\prime}\right), A\left(\mu_{1}\left(x^{\prime}\right), \ldots, \mu_{n}\left(x^{\prime}\right)\right)\right) \leq \\
\leq \sup _{x^{\prime} \in X} T\left(E_{2}\left(x, x^{\prime}\right), A\left(\mu_{1}\left(x^{\prime}\right), \ldots, \mu_{n}\left(x^{\prime}\right)\right)\right)= \\
=\tilde{A}_{E_{2}, T}\left(\mu_{1}, \ldots, \mu_{n}\right)(x) .
\end{gathered}
$$

It is true that if $E_{1} \leq E_{2}$, then

$$
\begin{gathered}
\tilde{A}_{E_{1}, \vec{T}}\left(\mu_{1}, \ldots, \mu_{n}\right)(x)= \\
=\inf _{x^{\prime} \in X} \vec{T}\left(E_{1}\left(x, x^{\prime}\right) \mid A\left(\mu_{1}\left(x^{\prime}\right), \ldots, \mu_{n}\left(x^{\prime}\right)\right) \geq\right. \\
\geq \inf _{x^{\prime} \in X} \vec{T}\left(E_{2}\left(x, x^{\prime}\right) \mid A\left(\mu_{1}\left(x^{\prime}\right), \ldots, \mu_{n}\left(x^{\prime}\right)\right)\right)= \\
=\tilde{A}_{E_{2}, \vec{T}}\left(\mu_{1}, \ldots, \mu_{n}\right)(x) .
\end{gathered}
$$

In some cases it is important to observe $T$-fuzzy equivalence relation $E$ on some particular $\alpha$-levels, $\alpha \in[0,1]$. For example, dealing with lower general aggregation operator, elements with low degrees of equivalence have major impact on the output of aggregation and we could obtain a distorted result. In such case it is reasonable to use $T$-fuzzy equivalence relation $E$ only above some $\alpha$-level, in that way ignoring elements with lower degrees of equivalence. We can consider $T$-fuzzy equivalence relation $E^{\alpha}$ :

$$
E^{\alpha}(x, y)= \begin{cases}E(x, y), & E(x, y) \geq \alpha \\ 0, & E(x, y)<\alpha\end{cases}
$$

or crisp equivalence relation $E_{\rho}^{\alpha}$ :

$$
E_{\rho}^{\alpha}(x, y)= \begin{cases}1, & E(x, y) \geq \alpha \\ 0, & E(x, y)<\alpha .\end{cases}
$$

For both cases the following implications hold:

$$
\begin{gathered}
\alpha_{1} \leq \alpha_{2} \Longrightarrow E^{\alpha_{1}} \geq E^{\alpha_{2}}, \\
\alpha_{1} \leq \alpha_{2} \Longrightarrow E_{\rho}^{\alpha_{1}} \geq E_{\rho}^{\alpha_{2}}
\end{gathered}
$$

for all $\alpha_{1}, \alpha_{2} \in[0,1]$. Thereby if $E^{\alpha_{1}} \geq E^{\alpha_{2}}$, then $\tilde{A}_{E^{\alpha_{1}}, T} \geq \tilde{A}_{E^{\alpha_{2}}, T}$ and $\tilde{A}_{E^{\alpha_{1}, \vec{T}}} \leq \tilde{A}_{E^{\alpha_{2}}, \vec{T}}$, as well as if $E_{\rho}^{\alpha_{1}} \geq E_{\rho}^{\alpha_{2}}$, then $\tilde{A}_{E_{\rho}^{\alpha_{1}}, T} \geq \tilde{A}_{E_{\rho}^{\alpha_{2}}, T}$ and $\tilde{A}_{E_{\rho}^{\alpha_{1}}, \vec{T}} \leq \tilde{A}_{E_{\rho}^{\alpha_{2}}, \vec{T}}$.

As we will see later, the properties described in this section are important, when we are dealing with approximate systems, based on upper and lower general aggregation operators.

\section{Approximate systems induced by upper and lower general aggregation operators}

The concept of an $\mathbb{M}$-approximate system was first introduced in [17] and further studied in [5, 7, 18]. This concept provides an alternative view on the relations between fuzzy sets and rough sets. This tool gives a framework allowing to generalize these theories.

In the context of $\mathbb{M}$-approximate systems two lattices play the fundamental role. The first one is an infinitely distributive complete lattice

$$
\mathbb{L}=\left(\mathbb{L}, \leq_{\mathbb{L}}, \wedge_{\mathbb{L}}, \vee_{\mathbb{L}}\right) .
$$

The bottom and the top elements of $\mathbb{L}$ are $0_{\mathbb{L}}$ and $1_{\mathbb{L}}$, respectively. The second lattice is denoted by $\mathbb{M}=\left(\mathbb{M}, \leq_{\mathbb{M}}, \wedge_{\mathbb{M}}, \vee_{\mathbb{M}}\right)$ and is assumed to be the complete lattice. The bottom and the top elements of $\mathbb{M}$ are $0_{\mathbb{M}}$ and $1_{\mathbb{M}}$, respectively. 
Definition 6.1 An upper $\mathbb{M}$-approximate operator on $\mathbb{L}$ is a mapping $u: \mathbb{L} \times \mathbb{M} \rightarrow \mathbb{L}$ such that

(1u) $u\left(0_{\mathbb{L}}, \alpha\right)=0_{\mathbb{L}}$ for all $\alpha \in \mathbb{M}$;

(2u) $a \leq u(a, \alpha), \quad \forall a \in \mathbb{L}$ for all $\alpha \in \mathbb{M}$;

(3u) $u(a \vee b, \alpha)=u(a, \alpha) \vee u(b, \alpha)$ for all $a, b \in \mathbb{L}$ and for all $\alpha \in \mathbb{M}$;

(4u) $u(u(a, \alpha), \alpha)=u(a, \alpha)$ for all $a \in \mathbb{L}$ and for all $\alpha \in \mathbb{M}$;

(5u) $\alpha \leq \beta, \alpha, \beta \in \mathbb{M} \Longrightarrow u(a, \alpha) \leq u(a, \beta)$ for all $\alpha \in \mathbb{L}$

(6u) if $0_{\mathbb{M}} \neq 1_{\mathbb{M}}$, then $u\left(a, 0_{\mathbb{M}}\right)=a$ for all $a \in \mathbb{L}$.

Definition 6.2 A lower $\mathbb{M}$-approximate operator on $\mathbb{L}$ is a mapping $l: \mathbb{L} \times \mathbb{M} \rightarrow \mathbb{L}$ such that

(1l) $l\left(1_{\mathbb{L}}, \alpha\right)=1_{\mathbb{L}}$ for all $\alpha \in \mathbb{M}$;

(2l) $a \geq l(a, \alpha), \quad \forall a \in \mathbb{L}$ for all $\alpha \in \mathbb{M}$;

(3l) $l(a \wedge b, \alpha)=l(a, \alpha) \wedge l(b, \alpha)$ for all $a, b \in \mathbb{L}$ and for all $\alpha \in \mathbb{M}$;

(4l) $l(l(a, \alpha), \alpha)=l(a, \alpha)$ for all $a \in \mathbb{L}$ and for all $\alpha \in \mathbb{M}$;

(5l) $\alpha \leq \beta, \alpha, \beta \in \mathbb{M} \Longrightarrow l(a, \alpha) \geq l(a, \beta)$ for all $\alpha \in \mathbb{L}$

(6l) if $0_{\mathbb{M}} \neq 1_{\mathbb{M}}$, then $l\left(a, 0_{\mathbb{M}}\right)=a$ for all $a \in \mathbb{L}$.

Definition 6.3 A triple $(\mathbb{L}, u, l)$, where

$$
u: \mathbb{L} \times \mathbb{M} \rightarrow \mathbb{L} \text { and } l: \mathbb{L} \times \mathbb{M} \rightarrow \mathbb{L}
$$

are upper and lower $\mathbb{M}$-approximate operators on $\mathbb{L}$, is called an $\mathbb{M}$-approximate system.

There are several works where some particular constructions of $\mathbb{M}$-approximate systems are presented $[7,18]$. In this paper we recall approximate systems induced by upper and lower general aggregation operators.

First, we describe the lattice $\mathbb{L}$ of all general aggregation operators:

$\mathbb{L}=\left\{\tilde{A}: \bigcup_{n}\left([0,1]^{X}\right)^{n} \rightarrow[0,1]^{X} \mid \tilde{A}\right.$ is a gen. agop $\}$.

The order $\leq_{\mathbb{L}}$ on $\mathbb{L}$ is defined as follows: for all $\mu_{1}, \ldots, \mu_{n} \in[0,1]^{X}$

$$
\tilde{A}_{1}\left(\mu_{1}, \ldots, \mu_{n}\right) \leq_{\mathbb{L}} \tilde{A}_{2}\left(\mu_{1}, \ldots, \mu_{n}\right) \Longleftrightarrow
$$

$\Longleftrightarrow \tilde{A}_{1}\left(\mu_{1}, \ldots, \mu_{n}\right)(x) \leq \tilde{A}_{2}\left(\mu_{1}, \ldots, \mu_{n}\right)(x), x \in X$.

The supremum $\tilde{A}_{1} \vee_{\mathbb{L}} \tilde{A}_{2}$ and infimum $\tilde{A}_{1} \wedge_{\mathbb{L}} \tilde{A}_{2}$ of two elements $\tilde{A}_{1}, \tilde{A}_{2} \in \mathbb{L}$ are defined for all $\mu_{1}, \ldots, \mu_{n} \in[0,1]^{X}$ and $x \in X$ respectively by

$$
\begin{gathered}
\left(\tilde{A}_{1} \vee \mathbb{L} \tilde{A}_{2}\right)\left(\mu_{1}, \ldots, \mu_{n}\right)(x)= \\
=\tilde{A}_{1}\left(\mu_{1}, \ldots, \mu_{n}\right)(x) \vee \tilde{A}_{2}\left(\mu_{1}, \ldots, \mu_{n}\right)(x) \text { and } \\
\left(\tilde{A}_{1} \wedge_{\mathbb{L}} \tilde{A}_{2}\right)\left(\mu_{1}, \ldots, \mu_{n}\right)(x)= \\
=\tilde{A}_{1}\left(\mu_{1}, \ldots, \mu_{n}\right)(x) \wedge \tilde{A}_{2}\left(\mu_{1}, \ldots, \mu_{n}\right)(x) .
\end{gathered}
$$

The top and the bottom elements $1_{\mathbb{L}}$ and $0_{\mathbb{L}}$ respectively are

$$
1_{\mathbb{L}}\left(\mu_{1}, \ldots, \mu_{n}\right)= \begin{cases}\tilde{1}, & \exists i \in\{1, \ldots, n\} \mu_{i} \neq \tilde{0} \\ \tilde{0}, \quad \mu_{1}=\ldots=\mu_{n}=\tilde{0}\end{cases}
$$

and

$$
0_{\mathbb{L}}\left(\mu_{1}, \ldots, \mu_{n}\right)= \begin{cases}\tilde{0}, & \exists i \in\{1, \ldots, n\} \mu_{i} \neq \tilde{1} \\ \tilde{1}, & \mu_{1}=\ldots=\mu_{n}=\tilde{1}\end{cases}
$$

The lattice $\mathbb{M}$ of all $T$-fuzzy equivalence relations is described in the following way:

$\mathbb{M}=\{E: X \times X \rightarrow[0,1] \mid E$ is a T-fuzzy equiv. rel. $\}$.

The order $\leq_{\mathbb{M}}$ on $\mathbb{M}$ is defined as follows:

$E_{1} \leq_{\mathbb{M}} E_{2} \Longleftrightarrow E_{1}(x, y) \leq E_{2}(x, y)$ for all $x, y \in X$.

The top and the bottom elements $1_{\mathbb{M}}$ and $0_{\mathbb{M}}$ respectively are

$$
\begin{gathered}
1_{\mathbb{M}}(x, y)=1 \text { and } \\
0_{\mathbb{M}}(x, y)= \begin{cases}0, & x \neq y \\
1, & x=y\end{cases}
\end{gathered}
$$

for all $x, y \in X$.

The approximation operators

$$
u: \mathbb{L} \times \mathbb{M} \rightarrow \mathbb{L} \text { and } l: \mathbb{L} \times \mathbb{M} \rightarrow \mathbb{L}
$$

in this case are defined as follows:

$$
\begin{gathered}
(u(\tilde{A}, E))\left(\mu_{1}, \ldots, \mu_{n}\right)(x)= \\
=\sup _{x^{\prime} \in X} T\left(E\left(x, x^{\prime}\right), \tilde{A}\left(\mu_{1}, \ldots, \mu_{n}\right)\left(x^{\prime}\right)\right), \\
(l(\tilde{A}, E))\left(\mu_{1}, \ldots, \mu_{n}\right)(x)= \\
=\inf _{x^{\prime} \in X} \vec{T}\left(E\left(x, x^{\prime}\right) \mid \tilde{A}\left(\mu_{1}, \ldots, \mu_{n}\right)\left(x^{\prime}\right)\right) .
\end{gathered}
$$

Most of the conditions from Definitions 6.1 and 6.2 follow from the properties considered previously in this paper and could be easily shown. There are needs for an additional argumentation for conditions $(3 l),(4 l)$ and $(3 u),(4 u)$.

(31) We prove that

$$
l\left(\tilde{A}_{1} \wedge \tilde{A}_{2}, E\right)=l\left(A_{1}, E\right) \wedge l\left(\tilde{A}_{2}, E\right)
$$

for all $\tilde{A}_{1}, \tilde{A}_{2} \in \mathbb{L}$ and for all $E \in \mathbb{M}$. For all $\mu_{1}, \ldots, \mu_{n} \in[0,1]^{X}$ and for all $x \in X$ it holds

$$
\begin{gathered}
\left(l\left(\tilde{A}_{1} \wedge_{\mathbb{L}} \tilde{A}_{2}, E\right)\right)\left(\mu_{1}, \ldots, \mu_{n}\right)(x)= \\
=\inf _{x^{\prime} \in X} \vec{T}\left(E\left(x, x^{\prime}\right) \mid\left(\tilde{A}_{1} \wedge_{\mathbb{L}} \tilde{A}_{2}\right)\left(\mu_{1}, \ldots, \mu_{n}\right)\left(x^{\prime}\right)\right)= \\
=\inf _{x^{\prime} \in X}\left[\vec{T}\left(E\left(x, x^{\prime}\right) \mid \tilde{A}_{1}\left(\mu_{1}, \ldots, \mu_{n}\right)\left(x^{\prime}\right)\right) \wedge\right. \\
\left.\wedge \vec{T}\left(E\left(x, x^{\prime}\right) \mid \tilde{A}_{2}\left(\mu_{1}, \ldots, \mu_{n}\right)\left(x^{\prime}\right)\right)\right]= \\
=\left(\inf _{x^{\prime} \in X} \vec{T}\left(E\left(x, x^{\prime}\right) \mid \tilde{A}_{1}\left(\mu_{1}, \ldots, \mu_{n}\right)\left(x^{\prime}\right)\right)\right) \wedge \\
\wedge\left(\inf _{x^{\prime} \in X} \vec{T}\left(E\left(x, x^{\prime}\right) \mid \tilde{A}_{2}\left(\mu_{1}, \ldots, \mu_{n}\right)\left(x^{\prime}\right)\right)\right)= \\
=\left(l\left(\tilde{A}_{1}, E\right)\right)\left(\mu_{1}, \ldots, \mu_{n}\right)(x) \wedge \\
\wedge\left(l\left(\tilde{A}_{2}, E\right)\right)\left(\mu_{1}, \ldots, \mu_{n}\right)(x) .
\end{gathered}
$$


(4l) We show that

$$
l(l(\tilde{A}, E), E)=l(\tilde{A}, E)
$$

for all $\tilde{A} \in \mathbb{L}$ and for all $E \in \mathbb{M}$. In order to prove this property, we will show that inequalities

$$
\begin{aligned}
& l(l(\tilde{A}, E), E) \leq l(\tilde{A}, E), \\
& l(l(\tilde{A}, E), E) \geq l(\tilde{A}, E)
\end{aligned}
$$

hold. The first inequality holds by property $(2 l)$. Let us prove that for all $\mu_{1}, \ldots, \mu_{n} \in[0,1]^{X}$ and for all $x \in X$

$$
\begin{gathered}
(l(l(\tilde{A}, E), E))\left(\mu_{1}, \ldots, \mu_{n}\right)(x) \geq \\
\geq(l(\tilde{A}, E))\left(\mu_{1}, \ldots, \mu_{n}\right)(x) .
\end{gathered}
$$

By the definitions we have

$$
\begin{gathered}
(l(l(\tilde{A}, E), E))\left(\mu_{1}, \ldots, \mu_{n}\right)(x)= \\
=\inf _{x^{\prime} \in X} \vec{T}\left(E\left(x, x^{\prime}\right) \mid(l(\tilde{A}))\left(\mu_{1}, \ldots, \mu_{n}\right)\left(x^{\prime}\right)\right) \text { and } \\
\vec{T}\left(E\left(x, x^{\prime}\right) \mid(l(\tilde{A}))\left(\mu_{1}, \ldots, \mu_{n}\right)\left(x^{\prime}\right)\right)= \\
=\sup \left\{\alpha \in[0,1] \mid T\left(\alpha, E\left(x, x^{\prime}\right)\right) \leq\right. \\
\left.\leq(l(\tilde{A}, E))\left(\mu_{1}, \ldots, \mu_{n}\right)\left(x^{\prime}\right)\right\} .
\end{gathered}
$$

Taking $\alpha=(l(\tilde{A}, E))\left(\mu_{1}, \ldots, \mu_{n}\right)(x)$, we obtain

$$
\begin{aligned}
& T\left((l(\tilde{A}, E))\left(\mu_{1}, \ldots, \mu_{n}\right)(x), E\left(x, x^{\prime}\right)\right) \leq \\
& \left.\leq(l(\tilde{A}, E))\left(\mu_{1}, \ldots, \mu_{n}\right)\left(x^{\prime}\right)\right\},
\end{aligned}
$$

which holds for all $x, x^{\prime} \in X$ by the fact, that $(l(\tilde{A}, E))\left(\mu_{1}, \ldots, \mu_{n}\right)$ is extensional fuzzy set with respect to $E$. It means that for all $x, x^{\prime} \in X$

$$
\begin{gathered}
\vec{T}\left(E\left(x, x^{\prime}\right) \mid(l(\tilde{A}, E))\left(\mu_{1}, \ldots, \mu_{n}\right)\left(x^{\prime}\right)\right) \geq \\
\geq(l(\tilde{A}, E))\left(\mu_{1}, \ldots, \mu_{n}\right)(x)
\end{gathered}
$$

and thus

$$
\begin{gathered}
\inf _{x^{\prime} \in X} \vec{T}\left(E\left(x, x^{\prime}\right) \mid(l(\tilde{A}, E))\left(\mu_{1}, \ldots, \mu_{n}\right)\left(x^{\prime}\right)\right) \geq \\
\geq(l(\tilde{A}, E))\left(\mu_{1}, \ldots, \mu_{n}\right)(x) .
\end{gathered}
$$

Properties $(3 u),(4 u)$ for upper approximation operator $u$ could proved analogously to the previous case.

\section{Acknowledgments}

This work has been supported by the European Social Fund within the project 2013/0024/1DP/1.1.1.2.0/13/APIA/VIAA/045.

\section{References}

[1] T. Calvo, G. Mayor, R. Mesiar, Aggregation Operators, Physica-Verlag, Heidelberg, 2002.

[2] J.L. Castro, F. Klawonn, Similarity in fuzzy reasoning, Mathware \& Soft Computing, 2:197-228, 1996.

[3] M. Detyniecki, Fundamentals on Aggregation Operators, Berkeley, 2001.

[4] D. Dubois, H. Prade, Rough fuzzy sets and fuzzy rough sets, Inernat. J. General Systems 17(23):191-209, 1990.

[5] A. Eļkins, S.-E. Han, A. Šostak, Variable-range approximate systems induced by many-valued L-relations. In proceedings of the 15th International Conference IPMU 2014, Communications in Computer and Information Science 444(3), pages 41-50, Springer-Verlag, 2014.

[6] M. Grabisch, J.-L. Marichal, R. Mesiar, E. Pap, Aggregation Functions, Cambridge University Press, 2009.

[7] S.-E. Han, I.S. Kim, A. Šostak, On approximatetype systems generated by L-relations, Inf. Sci., 281:8-20, 2014.

[8] J. Jacas, J. Recasens, Fixed points and generators of fuzzy relations, J. Math. Anal. Appl. 186:21-29, 1994.

[9] J. Jacas, J. Recasens, Fuzzy T-transitive relations: eigenvectors and generators, Fuzzy Sets and Systems, 72:147-154, 1995.

[10] E.P. Klement, R. Mesiar, E. Pap, Triangular Norms, Kluwer Academic Publishers, Dordrecht, 2000.

[11] G. Mattioli, J. Recasens, Comparison of different algorithms of approximation by extensional fuzzy sets. In proceedings of the 7 th $I n$ ternational Summer School on Aggregation Operators, Advances in Intelligent Systems and Computing 228, pages 307-317, Springer-Verlag, 2013.

[12] N.N. Morsi, M.M. Yakout, Axiomatics for fuzzy rough sets, Fuzzy Sets and Systems, 100:327-342, 1998.

[13] P. Orlovs, O. Montvida, S. Asmuss, A choice of bilevel linear programming solving parameters: factoraggregation approach. In proceedings of the 8th conference of the European Society for Fuzzy Logic and Technology, Advances in Intelligent Systems Research 32, pages 489-496, 2013.

[14] P. Orlovs, O. Montvida, S. Asmuss, An analysis of bilevel linear programming solving parameters based on factoraggregation approach. In proceedings of the 7th International Summer School on Aggregation Operators, Advances in Intelligent Systems and Computing 228, pages 345-354, Springer-Verlag, 2013.

[15] P. Orlovs, S. Asmuss, Upper and lower generalized factoraggregations based on fuzzy equivalence relation. In proceedings of the 2014 IEEE International Conference on Fuzzy Systems 
(FUZZ-IEEE 2014), pages 1772-1777, 2014.

[16] P. Orlovs, S. Asmuss, On extensional fuzzy sets generated by factoraggregation. In proceedings of the 15th International Conference IPMU 2014, Communications in Computer and Information Science 444(3), pages 317-326, SpringerVerlag, 2014.

[17] A. Šstak, On approximative operators and approximative system. In proceedings of Congress of the International Fuzzy System Association (IFSA 2009), pages 1061-1066, 2009.

[18] A. Sostak, Towards the theory of Mapproximate systems: Fundamentals and examples, Fuzzy Sets and Systems, 161:2440-2461, 2014.

[19] A. Takaci, General aggregation operators acting on fuzzy numbers induced by ordinary aggregation operators, Novi Sad J. Math., 33(2):6776, 2003.

[20] Y.Y. Yao, A comparative study of fuzzy sets and rough sets, Inf. Sci., 109:227-242, 1998.

[21] L. Zadeh, Similarity relations and fuzzy ordering, Inf. Sci., 3(2):177-200, 1971. 